\title{
Effect of Addition of Different Levels of Commercial Baker's Yeast to Concentrate Diet of Multiparous Mid-Lactation Holstein Friesian Cows on Rumen Fermentation Characteristics
}

\author{
Waleed Ahmed Mohammed \\ Ali Ameen Saeed \\ Al-Qasim Green University, College of Agriculture, Department of Animal \\ Production \\ aliameensaeed@Yahoo.com
}

ARTICLE INFO

Submission date: $6 / 11 / 2018$

Acceptance date: $29 / 11 / 2018$

Publication date: $10 / 3 / 2019$

\begin{abstract}
This study was conducted in Grand station of Diwania/ Taj Al-Nahrain company- Al-Qadisiya Province for 84 days to investigate the effect of level of addition of commercial baker's yeast Saccharomyces cerevisiae,0, 15 and $30 \mathrm{~g} / \mathrm{cow} / \mathrm{day}$ to concentrate diet on rumen fermentation characteristics in German Holstein Friesian lactating cows. Nine multiparous Holstein cows in their mid-lactation were used. Daily feeding program applied in that station included offering $1 \mathrm{~kg}$ of concentrate diet per 3-4 kg milk produced divided into morning and evening meals, $10 \mathrm{~kg}$ of green forage, $10 \mathrm{~kg}$ whole plant corn silage and free choice of rice straw. Silage was characterized with accepted quality and fermentation parameters, values of $\mathrm{pH}$, concentrations of ammonia nitrogen $\left(\mathrm{NH}_{3}-\right.$ $\mathrm{N}$ ) and total volatile fatty acids (TVFA) were, 3.5, $0.98 \%$ of total nitrogen and $2.55 \mathrm{mM} / \mathrm{DM}$ respectively. Results revealed that addition of yeast at level of 15 and $30 \mathrm{~g} / \mathrm{cow} /$ day reduced $(\mathrm{P}<0.01)$ concentration of $\mathrm{NH}_{3}-\mathrm{N}$ from 18.14 to 13.92 and $14.21 \mathrm{mg} / 100 \mathrm{ml}$ respectively. Significant $(\mathrm{P}<0.01)$ increase in concentration of TVFA from 7.32 to $9.39 \mathrm{mM} / 100 \mathrm{ml}$ was also observed due to addition of yeast at level of $15 \mathrm{~g} / \mathrm{cow} /$ day. However, ruminal $\mathrm{pH}$ values were not affected by addition of yeast.
\end{abstract}

Key words: Baker's yeast, dairy cow, milk, rumen fermentation, blood parameters 


\section{تأثير إظافة مستويات مختلفة من خميرة الخبز التجارية الذ العليقة المركزة لأبقار} الهولشتاين فريزيان متعددة المواسم فيخ المرحلة الوسطية من الاءنتاج على خ خصائر

$$
\begin{aligned}
& \text { تخمرات الكرشر } \\
& \text { وليد احمد محمد علي أمين سعيد } \\
& \text { كلية الزراعة-جامعة القاسم الخضراء -قسم الإنتاج الحيواني }
\end{aligned}
$$

الخلاصة

أجريت الدراسة في محطة أبقار الديوانية الكبرى/شركة تاج النهرين- محافظة الديوانية لمدة 84 يوما للتحري عن تأثير مستوى

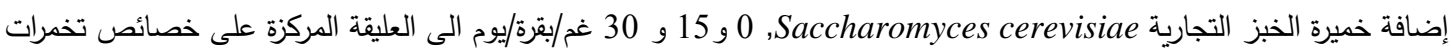

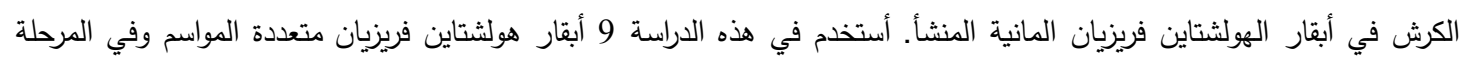

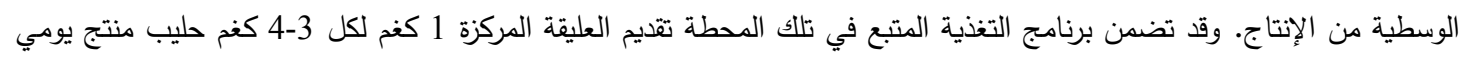

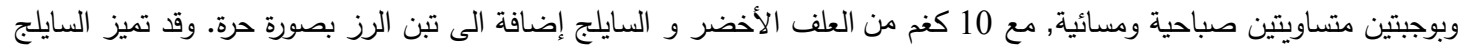

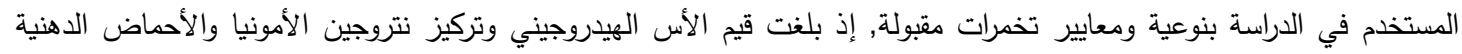

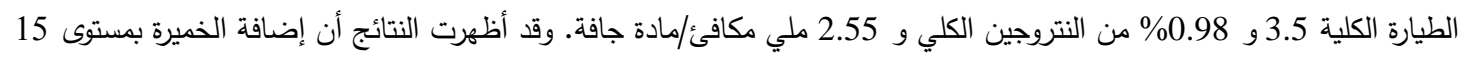

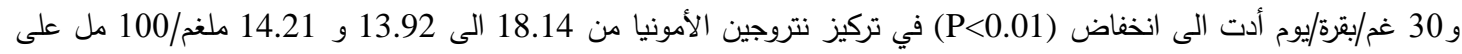

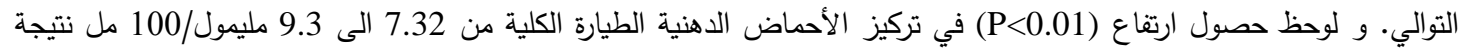
لإضافة الخميرة بمستوى 15 غم/بقرة/يوم. إلا ان قيم الأس الهيدروجيني في الكرش لم تتأثر بمستوى إضافة الخميرة.

$$
\text { الكلمات الدالة: خميرة الخبز, أبقار الحليب, الحليب, تخمرات الكرش, معايير الدم }
$$

\section{المقدمة}

مما لاشك فيه أن قطاع الثروة الحيوانية في العراق قد شهد تراجعا ملحوظا منذ عقد الثمانينات حيث انخفضت أعداد الحيوانات الزراعية ومنها الأبقار والجاموس كما يثير الى ذلك الاحصاء الذي أعدته المنظمة العربية للتمية الزراعية [1]. ونظرا لانحسار مساحة الأراضي الصالحة للزراعة وانخفاض إنتاج محاصيل العلف

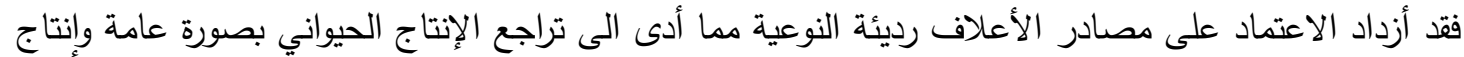

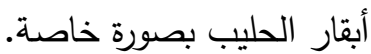

ويتطلب تحسين إنتاج وتركيب الحليب في الأبقار تعزيز الاستفادة من المصادر العلفية المتوفرة. وقد أستخدم لتأمين ذلك الهدف العديد من المكملات والإضافات الغذائية مثل المضادات الحيوية. إلا إن القلق المتزايد من استعمال تلك المركبات أدى الى الاهتمام باستخدام المعززات الحيوية كالخميرة والبكتريا وتفضيلها

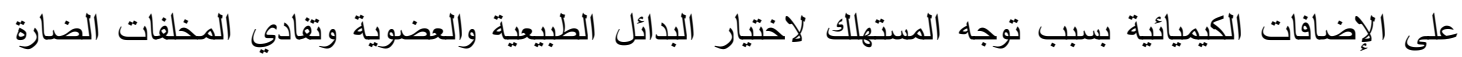

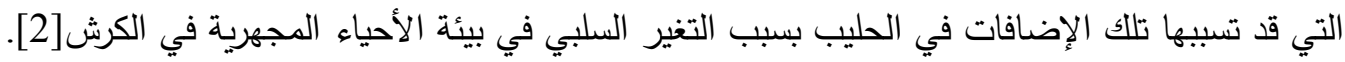
وتساعد خلايا الخميرة في زيادة الفعالية الميكروبية في الكرش من خلال محتواها المرتفع من الفيتامينات

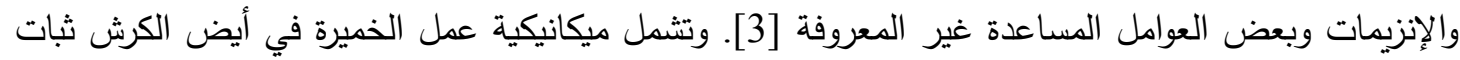

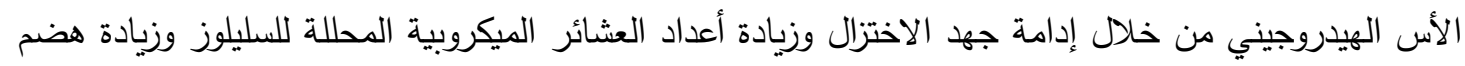
الألياف تبعا لللك والاستفادة من النشا والسكريات في خفض معدل إنتاج حامض اللاكتيك وتجنب حصول حموضة الكرش وتحرير الفيتامينات وعوامل النمو لتحفيز النشاط الميكروبي [4]. ونظرا لتوفر خميرة الخبز 
التجارية وأسعارها الواطئة فقد اجريت الدراسة الحالية للتحري عن تأثير مستوى اضافة تلك الخميرة على تخمرات الكرش في أبقار الهولشتاين فريزيان.

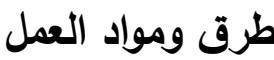

أجريت الدراسة في شركة تاج النهرين/محطة أبقار الديوانية الكبرى لمدة 84 يوما بضمنها 14 يوما

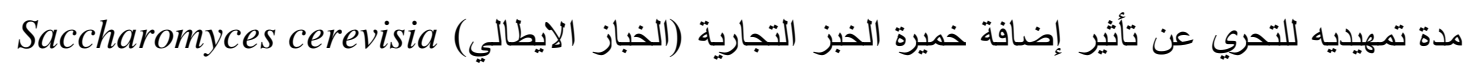

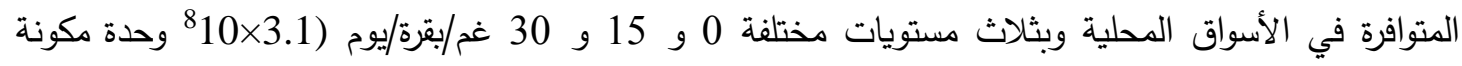

للسبورات/غم) على تخمرات الكرش في أبقار الهولشتاين فريزيان المانية المنشأ المرباة محليا.

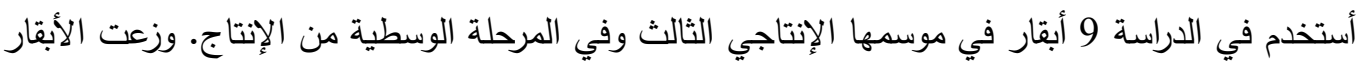

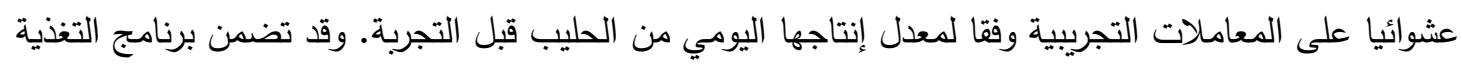
المتبع في تلك المحطة استخدام كل من العليقة المركزة والعلف الأخضر و السايلج إضافة الى تبن الرز. حضرت العليقة المركزة بخلط 40\% من العلف المركز (إنتاج الشركة المغذية المصرية) مع 60\% من من الذرة الصفراء. وقد روعي إضافة الخميرة الى العليقة المركزة موقعيا بمستوى 15 و 30 غم/لبقرة/يوم فيما عدت العليقة

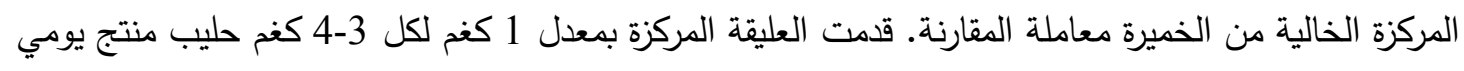
وبوجبتين متساويتين صباحية ومسائية. قدمت الأعلاف الخضراء بكمية بلغت حوالي 10 كغم/بقرة/يوم (حوالي 1.87 كغم مادة جافة). وقدم سايلج علف الذرة (إيراني المنشأ) بمعدل 10 كغم/بقرة/يوم (3.05 كغم مادة جافة). وقد تميز السايلج المستخدم في الدراسة بنوعية ومعايير تخمرات مقبولة, إذ بلغت قيم الأس الهيدروجيني وتركيز

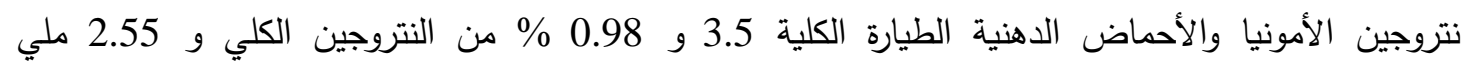
مكافئ/مادة جافة. أما العلف الخشن (تبن الرز) فقد قدم بصورة حرة. ويوضح جدول (1) الأنية التركيب الكيميائي للأعلاف المستخدمة في الدراسة.

جدول 1- التركيب الكيميائي للمواد العلفية المستخدمة في الدراسة (\%)

\begin{tabular}{|c|c|c|c|c|c|c|}
\hline \multicolumn{5}{|c|}{ \% من المادة الجافة } & \multirow[t]{2}{*}{ DM } & \multirow[t]{2}{*}{ المادة العلفية } \\
\hline NFE & $\mathrm{EE}$ & $\mathrm{CF}$ & $\mathrm{CP}$ & $\mathrm{OM}$ & & \\
\hline 63.9 & 3.75 & 5.8 & 7.55 & 81.46 & 91.67 & العلف المركز \\
\hline 65.61 & 3.19 & 16.25 & 4.40 & 89.45 & 18.70 & العلف الاخضر \\
\hline 53.88 & 2.78 & 29.76 & 3.03 & 89.45 & 30.50 & السايلج \\
\hline 37.91 & 1.45 & 35.13 & 2.73 & 77.22 & 92.25 & تبن الرز \\
\hline
\end{tabular}

أجريت التحليلات الكيميائية لنماذج العليقة المركزة والعلف الأخضر والسايلج والتبن وفقا الى طرائق [5]AOAC. أما خصائص تخمرات السايلج فقد قدرت في المستخلص المائي للسايلج الذي حضر بمزج 50 غم

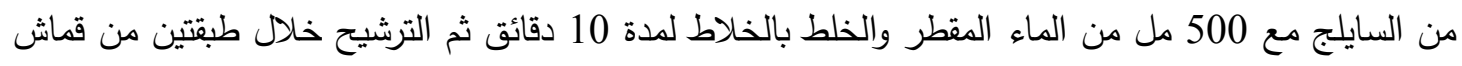
الململ وتمرير الراشح خلال ورقة ترشيح [6]. قسم الراشح على ثلاثة أقسام لتقدير الأس الهيدروجيني أولا فيما

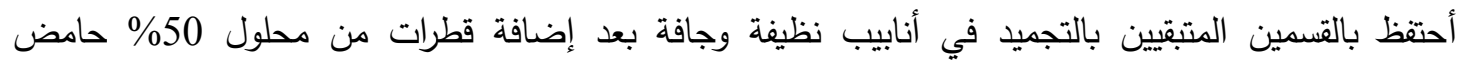
الكبريتيك لحين تقدير تركيز نتروجين الأمونيا والأحماض الدهنية الطيارة الكلية[7]. 
تم سحب سائل الكرش من الأبقار في اليوم الأخير من التجربة وبعد التغذية الصباحية باستخدام بلاستيكية خاصة. رشح السائل المسحوب من خلال أربعة طبقات من قماش الململ ثم قسم وحفظ الراشح بطريقة مماثلة لحفظ المستخلص المائي للسايلج. قدر الأس الهيدروجيني في المستخلص المائي للسايلج وسائل الكرش مباشرة بعد الترشيح وقبل الحفظ بالتحميض كما مبين أعلاه باستخدام جهاز Mi 180 Bench Meter بعد تعديله بالمحاليل المنظمة.

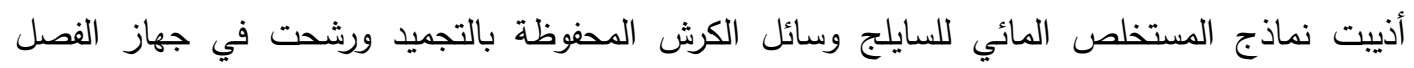
الكهربائي على 3000 دورة ولمدة 20 دقيقة. ثم تم تقدير تركيز نتروجين الأمونيا باستخدام طريقة التقطير بأوكسيد المغنيسيوم وذلك بوضع 0.5 مل من المستخلص أو سائل الكرش في أنبوبة الهضم الخاصة بجهاز

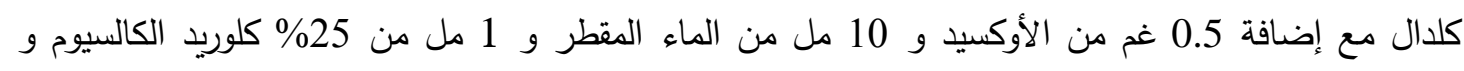

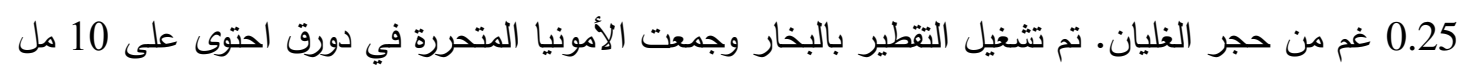

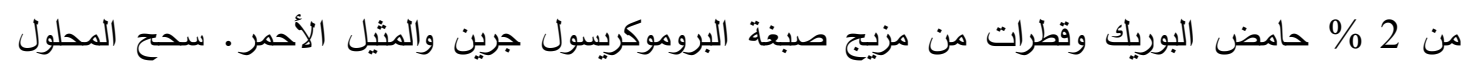

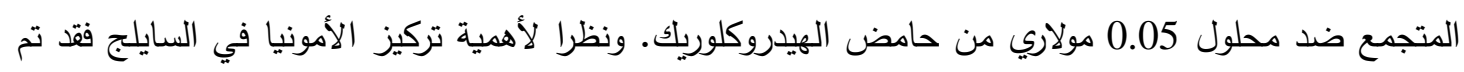

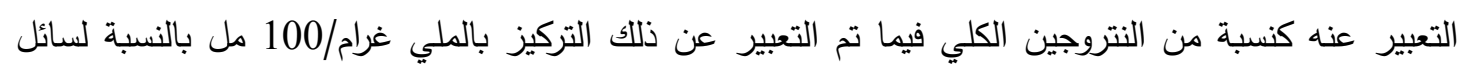
الكرش. أذيبت نماذج القسم الثالث من المستخلص المائي للسايلج وسائل الكرش المحفوظة بالتجميد ورشحت بطريقة

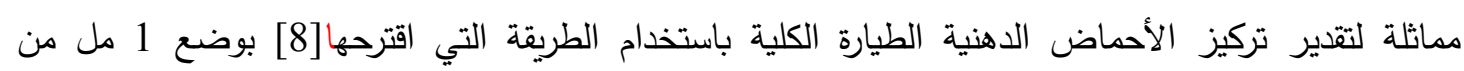

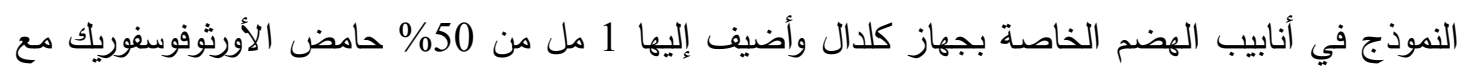

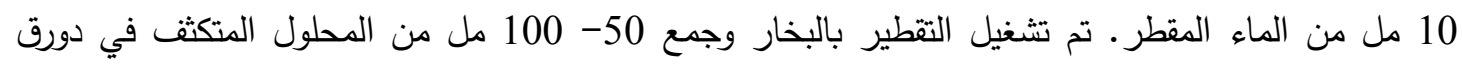

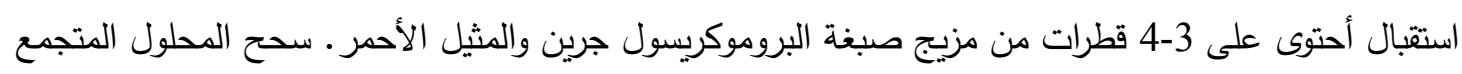
ضد محلول 0.1 عياري من هيدروكسيد الصوديوم. ونظرا لأهمية تركيز الأحماض الدهنية الطيارة في السايلج

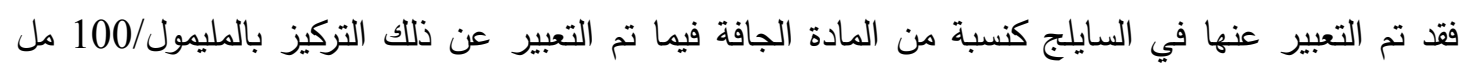
بالنسبة لسائل الكرش.

\section{النتائج وإلمناقشة}

تأثير مستوى إضافة خميرة الخبز التجارية على الأس الهيروجيني في الكرش:

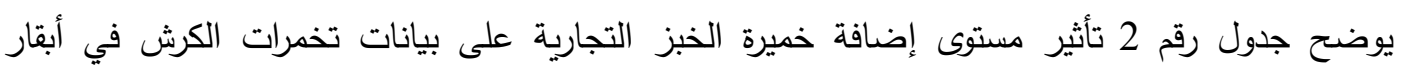

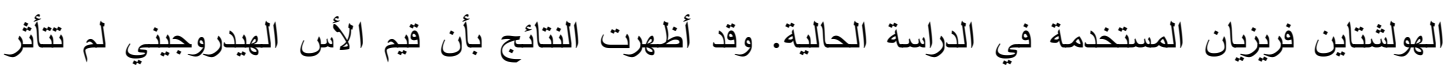

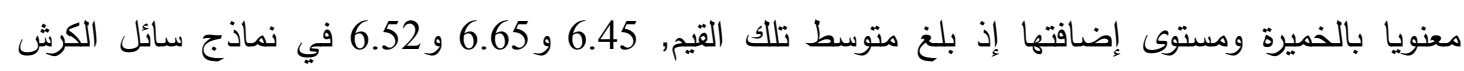

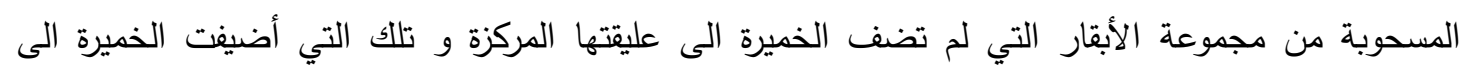
عليقتها بمستوى 15 و 30 غم/بقرة/يوم على التوالي. 
Journal of University of Babylon, Pure and Applied Sciences, Vol. (27), No. (1): 2019

جدول 2 - تأثير مستوى إضافة خميرة الخبز على تخمرات الكرش في أبقار الهولثتاين فريزيان (الوحدات حسب ظهورها في الجدول \pm الخطأ القياسي)

\begin{tabular}{|c|c|c|c|c|}
\hline \multicolumn{4}{|c|}{ مستوى الخميرة غم/بقرة/يوم } & \multirow[b]{2}{*}{ معيار التخمرات } \\
\hline المعنوية & 30 & 15 & 0 & \\
\hline م & $\begin{array}{c}6.52 \\
0.02 \pm\end{array}$ & $\begin{array}{c}6.65 \\
0.10 \pm\end{array}$ & $\begin{array}{c}6.45 \\
0.06 \pm\end{array}$ & الأس الهيدروجيني \\
\hline$* *$ & $\begin{array}{l}14.21^{b} \\
0.12 \pm\end{array}$ & $\begin{array}{l}13.92^{\mathrm{b}} \\
0.34 \pm\end{array}$ & $\begin{array}{l}18.14^{\mathrm{a}} \\
0.09 \pm\end{array}$ & نتروجين الأمونيا (ملغم/100 مل) \\
\hline$* *$ & $\begin{array}{c}7.52^{\mathrm{b}} \\
0.17 \pm\end{array}$ & $\begin{array}{l}9.39^{\mathrm{a}} \\
0.17 \pm\end{array}$ & $\begin{array}{l}7.32^{b} \\
0.63 \pm\end{array}$ & 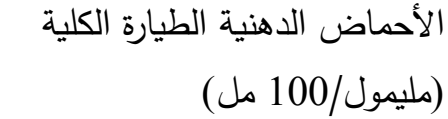 \\
\hline
\end{tabular}

المتوسطات التي تحمل حروفا مختلفة تختلف معنويا فيما بينها

غ م = غير معنوي

غياب التأثير المعنوي لإضافة الخميرة على الأس الهيدروجيني للكرش جاء منسجما مع نتائج العديد من

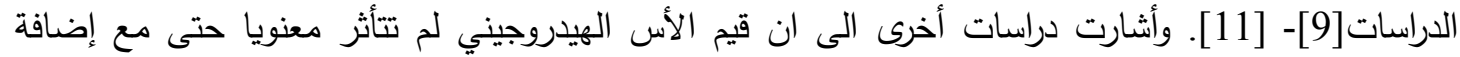
مستويات مختلفة من الخميرة الى علائق أبقار الحليب [12] -[13]. وقد سجلت قيم للأس الهيدروجيني في الكرش مقاربة للقيم التي أظهرتها نتائج الدراسة الحالية إذ بلغت تللك القيم 6.51 و 6.54 و 6.57 في نماذج سائل الكرش المسحوبة من أبقار الهولشتاين والجيكية الحمراء المغذاة

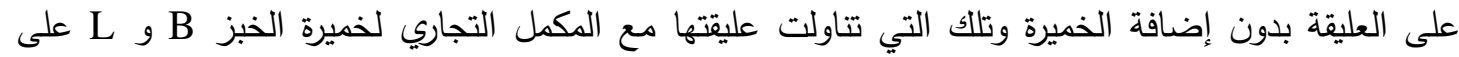
التوالي [14] • وكذللك القيم التي حصل عليها[15] في نماذج سائل الكرش للأبقار التي أكملت عليقتها بالخميرة

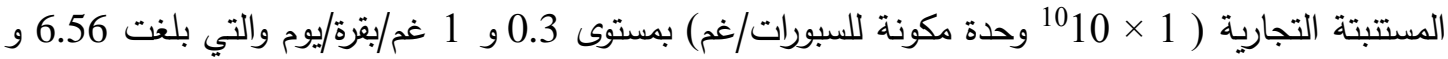
6.59 و 6.63 على التوالي. فيما أظهرت نتائج دراسات أخرى قيما أوطأ مما سجل في الدراسة الحالية, 5.15 فو و

6.15 في نماذج سائل الكرش المسحوبة من الأبقار في مجموعة المقارنة ومجموعة الإضافة على التوالي[16].

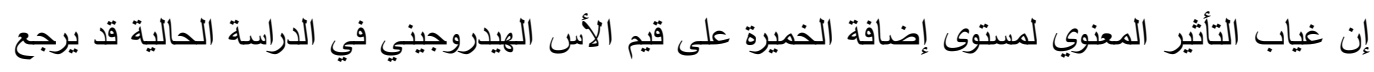

الى دور الخميرة في تتظيم تخمرات الكرش والدفع باتجاه حصول حالة من الاستقرار في قيم الأس الهيدروجيني

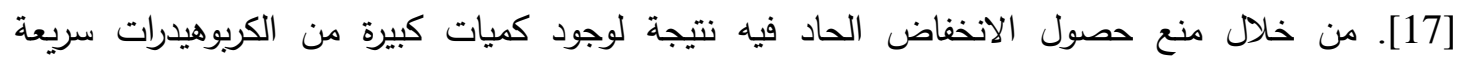
التخمر [18]. فقد لاحظ [19] أن وجود إضافة خميرة الخبز الى العليقة المركزة لأبقار الهولشتاين قد أسهم في كني

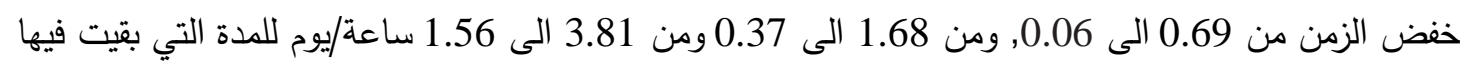

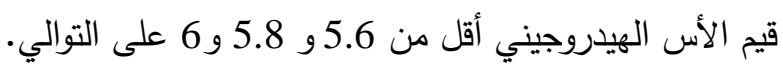
وقد يرجع تأثير خميرة الخبز المحدد لانخفاض الأس الهيدروجيني في الكرش الى الى انخفاض تركيز حامض اللاكتيك في الكرش[20]. من خلال زيادة نشاط البكتيريا الممثلة للحامض مثل كئل Selenomonas ruminantium لحامض اللاكتيك[23]. ويحدث ذلك من خلال تقليل توفر الكلوكوز لتخليق الحامض من قبل بكتيريا 
[15treptococcus bovis الهيدروجيني وتقليل التذبذب فيه وخلق حالة من الاستقرار في بيئة الكرش بتوفير الظروف الملائمة لنمو ونشاط

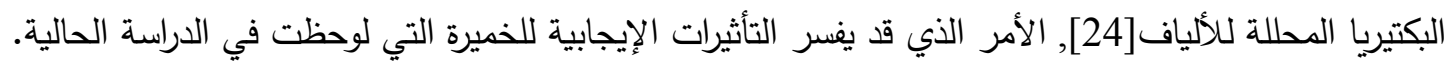
وعلى الرغم مما تقدم فإن عدم تأثر قيم الأس الهيدروجيني في الكرش بمستوى إضافة خميرة الخبز التجارية

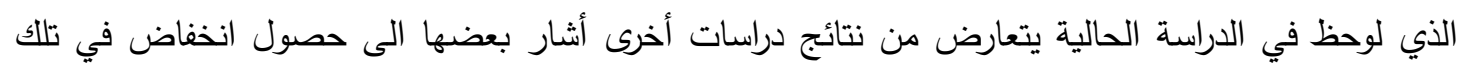

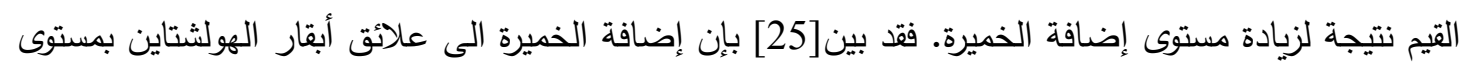

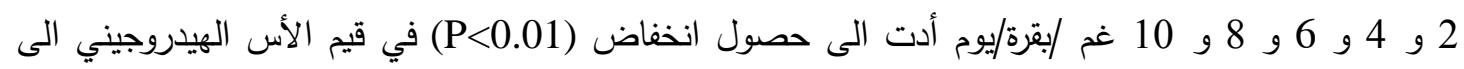

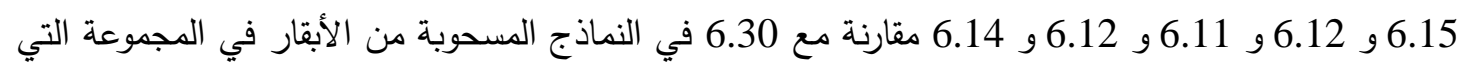
لم تضف الخميرة الى عليقتها. وفي دراسات أخرى سجلت قيم الأس الهيدروجيني ارتفاعا معنويا (P<0.01) [15] [16] [16]. وأوضح [19] أن إضافة خميرة الخبز الى عليقة أبقار الحليب أدت الى حصول زيادة معنوية 6.53 6.53 من 6.32 الى 6.01$)$

تأثير مستوى إضافة خميرة الخبز على تركيز نتروجين الأمونيا في الكرش:

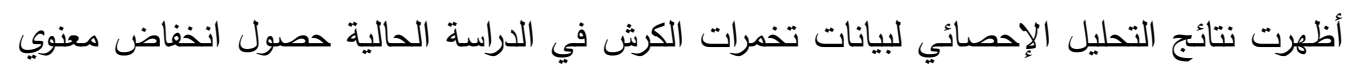
(P<0.01)

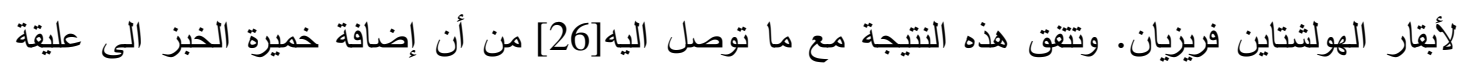

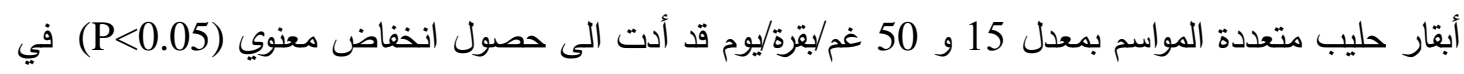

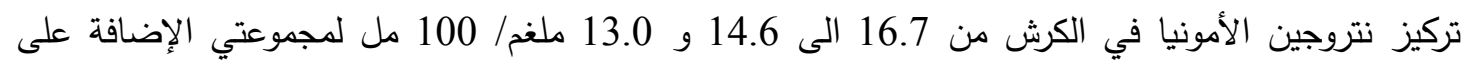
التوالي. في الدراسة الحالية أدت إضافة خميرة الخبز التجارية الى خفض ترنيل تركيز نتروجين الأمونيا معنويا من 18.14 في نماذج سائل الكرش المسحوبة من أبقار الهولشتاين فريزيان المغذاة على العليقة المركزة الخالية من الخميرة الى 13.92 و 14.21 ملغم/ 100 مل في النماذج المسحوبة من الأبقار المغذاة على العليقة التي أضيفت إليها الخميرة بمستوى 15 و 30 غم/بقرة/يوم على التوالي. وفي دراسة أخرى أكد [25] على تأثير مماثل لإضافة الخميرة على تركيز نتروجين الأمونيا في الكرش, التها.

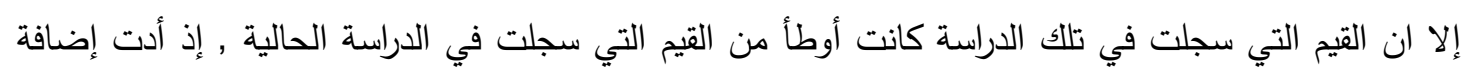

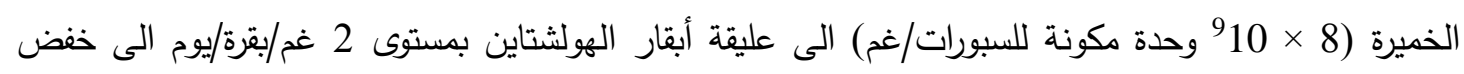

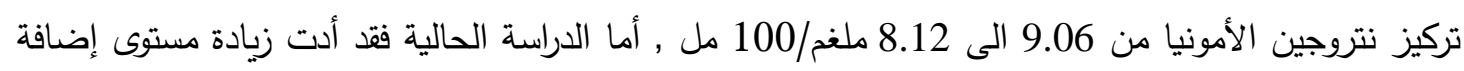

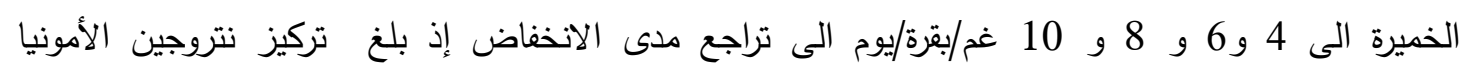

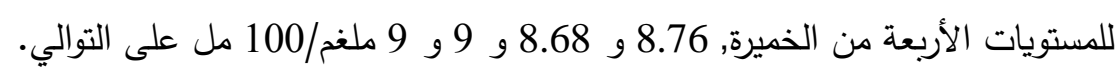

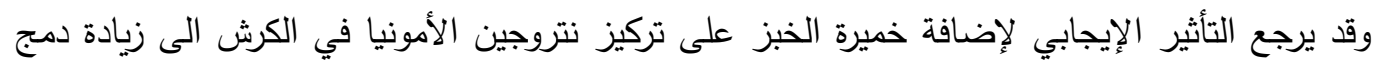
الأمونيا الناتجة من التحلل الميكروبي للبروتين الغذائي في الكرش في البروتين الميكروبي المخلق نتيجة لتركيز نتروجين لتعزيز

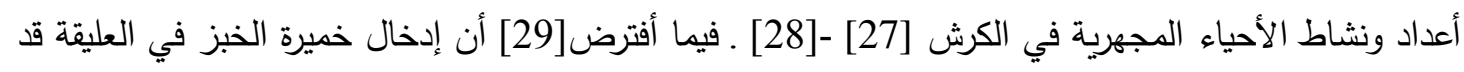
يؤدي الى تراجع عمليات تحلل البروتين الغذائي في الكرش نتيجة لتثبيط فعالية انزيم اليوريز الميكروبي. وسواء أدت الخميرة الى زيادة تخليق البروتين الميكروبي أو تتبيط التحلل البروتيني في الكرش فاني كان كلاهما سيسهم في

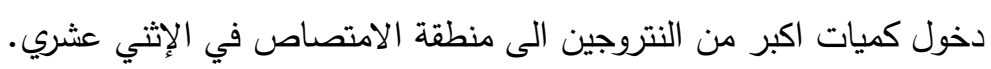


غير ان دور خميرة الخبز في تأمين الظروف الملائمة لنمو ونشاط البكتيريا المحللة للألياف الذي أجمعت عليه معظم الدراسات [30] - [32], يرجح أن تكون زيادة ادخال نتروجين الأمونيا في تركيب البروتين الميكروبي سببا لانخفاض تركيزها في الكرش نظرا لحاجة البكتيريا المحلة للألياف للأمونيا كمصدر للنتروجين. وعلى الرغم من الانخفاض المعنوي (P<0.01) في تركيز نتروجين الأمونيا الذي سجل في في الدراسة

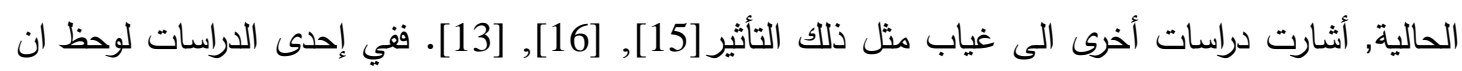
اضافة خميرة الخبز الىى عليقة أبقار الهولشتاين متعددة المواسم بمعدل 4 غم/بقرة/يوم (15×1019 وحدة مكونة

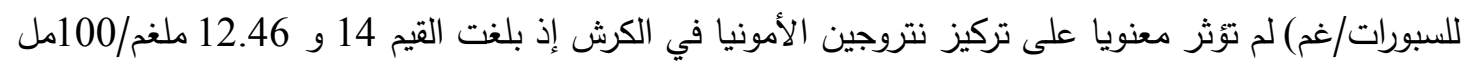

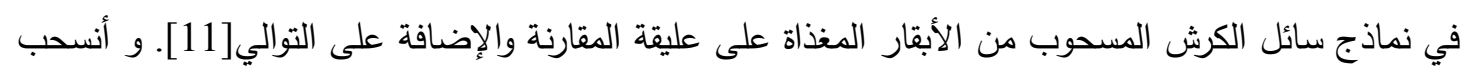
غياب تأثير الخميرة أيضا على مستوى الإضافة فقد أشار [12] الى ان زيادة مستوى إضافة المنتجات التجارية

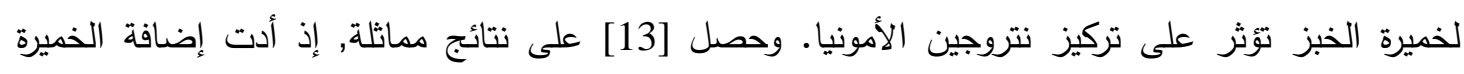

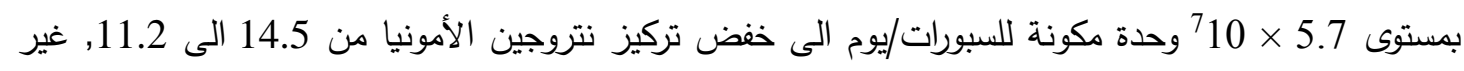
ان زيادة مستوى الإضافة الى 6 × 108 وحدة مكونة للسبورات/يوم وبنمط مماثل لما حصل في الدراسة الحالية

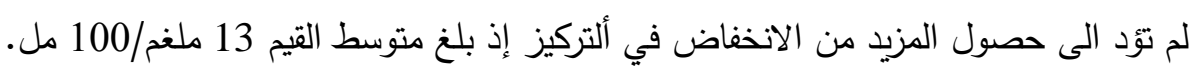

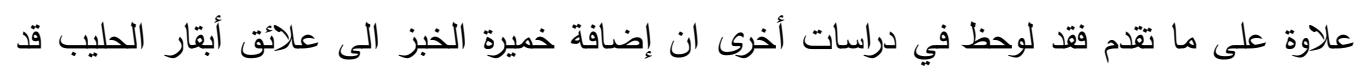

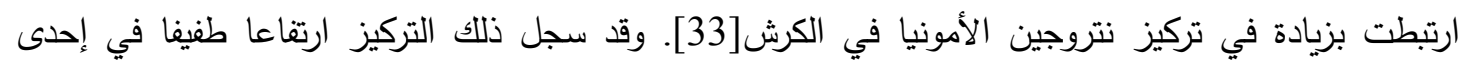

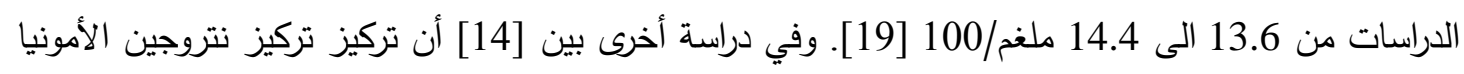

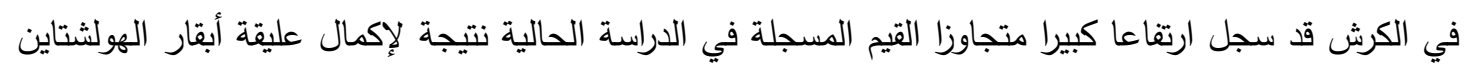

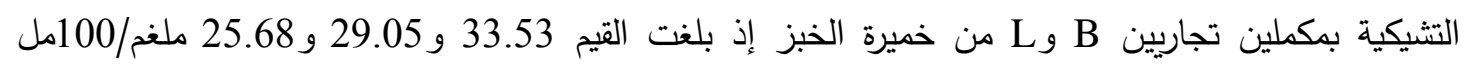
لمجموعة المقارنة ومجموعتي الإضافة على التوالي, وقد عزى أولئك الباحثون ارتفاع قيم تركيز نتروجين الأمونيا الى ارتفاع التتاول من البروتين الخام في العليقة وزيادة نسبة الجزء المتحلل منهـ.

تأثير مستوى إضافة خميرة الخبز على تركيز الأحماض الدهنية الطيارة الكلية في الكرش:

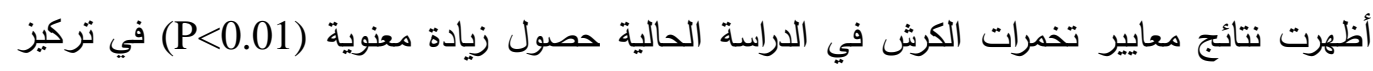
الأحماض الدهنية الطيارة في الكرش نتيجة لإضافة خميرة الخبز التجارية الى العليقة المركزة لأبقار الهولشتاين

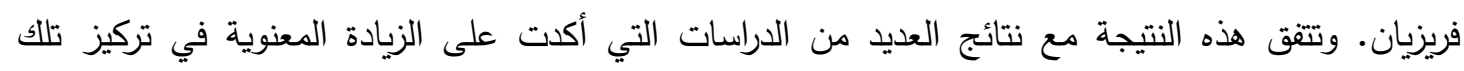

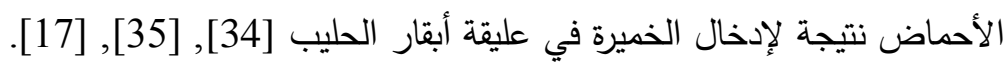
وقد اقتصر التأثير الإيجابي لإضافة خميرة الخبز في الدراسة الحالية على المستوى المنخفض (15) فم/يقرة/يوم) دون أن يلاحظ مثل ذلك التأثير عند إضافة الخميرة بالمستوى المرتفع (30 غم/يقرة/يوم), إذ ارتفع تركيز الأحماض الدهنية الطيارة (P<0.01) من 7.32 في نماذج سائل الكرش المسحوبة من مجموعة الابقار

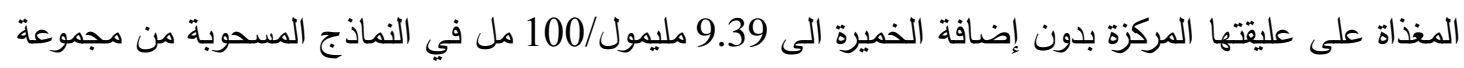

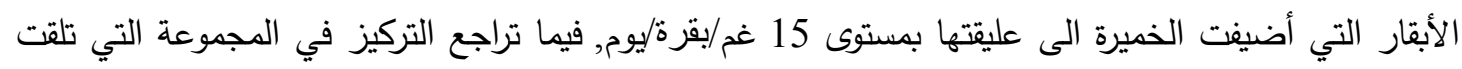

30 غم/بقرة/يوم من الخميرة ليقترب من القيم المسجلة في مجموعة المقارنة.

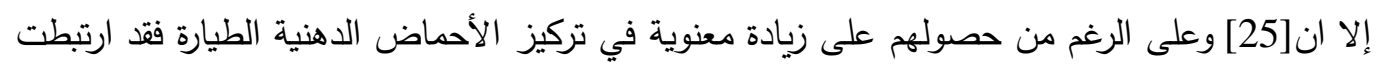
بنمط مغاير لنمط الزيادة المتحققة في الدراسة الحالية, إذ أدت زيادة مستوى إضافة خميرة الخبز الى على عليقة أبقار الهولشتاين, 2 و 4 و و8 و 10 غم/بقرة/يوم الى حصول زيادة خطية (P) (P) في تركيز الأحماض الدهنية 
الطيارة من 8.40 الى 10.42 و 11.10 و 11.58 و 12.10 و 12.76 مليمول/100 مل للمستويات الخمسة من الخميرة على التوالي.

وحصل [12] على نتائج مماثلة إذ أدت زيادة مستوى إضافة المنتجات التجارية لخميرة الخبز , 0 و 60 الخئ و 120 و 180 غم/يقرة/يوم الى عليقة أبقار الحليب في مرحلة الإنتاج الوسطية الى حصول زيادة خطية

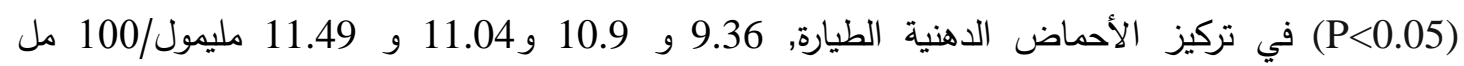

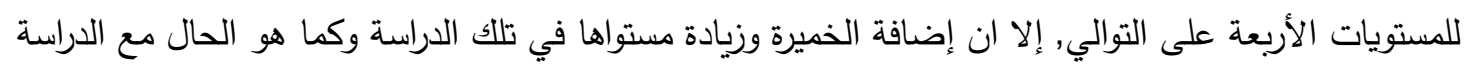
الحالية لم تؤثر على قيم الأس الهيدروجيني في الكرش. ومن المرجح ان ترجع الزيادة في تركيز الأحماض الدهنية نتيجة لإضافة الخميرة الى تحسن لتصن تخمرات

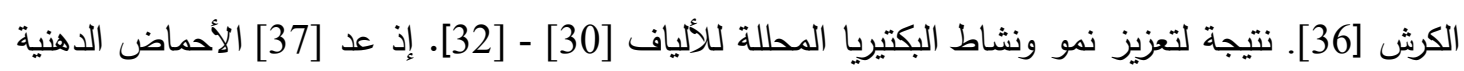
الطيارة من المكونات الطبيعية لمحتويات الكرش وتمثل الناتج النهائي لتخمر الكربوهيدرات بفعل الأحياء المجهرية وتسهم في تلبية (70\%) من احتياج الحيوان من الطاقة. وإزالة مجموعة الأمين من الأحماض الأمينية

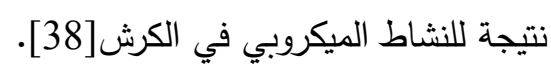
ولاحظ[39] أن زيادة أعداد البكتيريا المحلة للسليلوز ارتبطت بزيادة تركيز حامض الأسيتيك في الكرش. ويثكل ذلك الحامض الجزء الأكبر من الأحماض الدهنية الطيارة الكلية الناتج النهائي الرئيس لنشاط البكتيريا المحللة للسليلوز [40].

ويمكن أن تعزى الزيادة في تركيز الأحماض الدهنية الطيارة نتيجة لإضافة خميرة الخبز الى دورها في

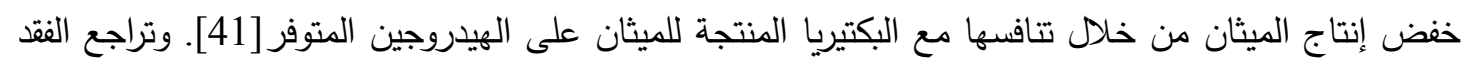
في الطاقة نتيجة لذلك مما يساعد في توفير كميات إضافية من الطاقة لتخليق الأحماض الدهنية الطيارة قصيرة

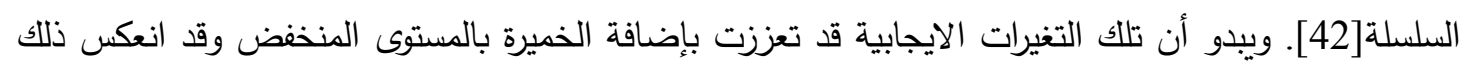
ايجابيا على إنتاج الحليب من قبل مجموعة أبقار الهولثتاين فريزيان التي أضيفت الخميرة الى عليقتها المركزة بمستوى 15 غم/بقرة/يوم وزيادة مستوى الدهن فيه. على الرغم من التحسن في إنتاج الأحماض الدهنية الطيارة الكلية في الكرش الذي أذي أظهرته نتائج الدراسة الحالية ودراسات عديدة أخرى ذكر [11] أن إضافة خميرة الخبز الى على عليقة أبقار الهولثتاين متعددة المواسم بمعدل 4 غم/بقرة/يوم (15×109 وحدة مكونة للسبورات/غم), لم تؤثر معنويا على تركيز الأحماض الدهنية الطيارة الكلية التي بلغت 8.51 و 9.02 مليمول/100مل لمجموعة المقارنة والإضافة على الفى التوالي. وفي دراسة

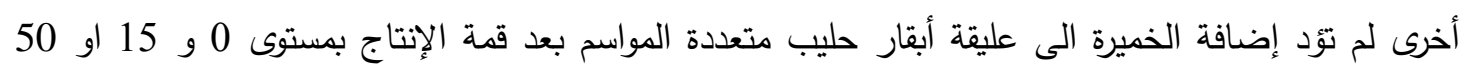

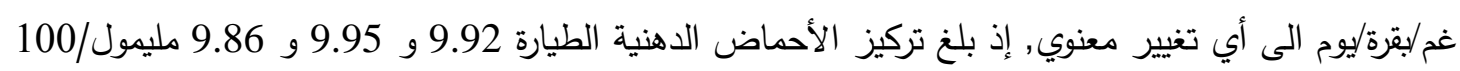
مل[26] فم

كما أوضح[13] أن إضافة الخميرة الى أبقار الحليب بمستوى 5.7 × 10 10 او 6 × 10 أ 8 وحدة مكونة للسبورات/يوم خميرة لم تؤثر على تركيز الأحماض الدهنية الطيارة الكلية التي بلغت, 6.86 و 6.39 و 7.71 × 7.

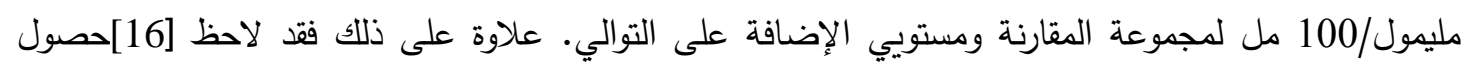

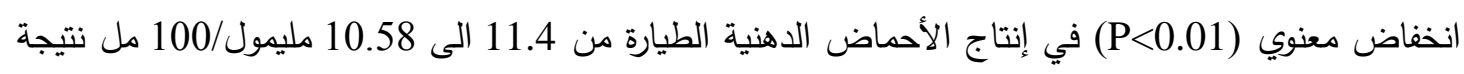

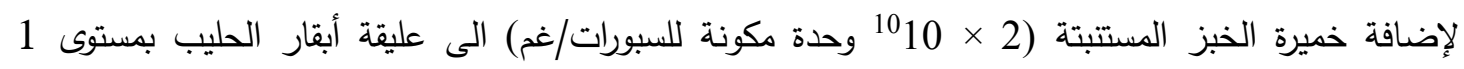
غم/بقرة/يوم. وحصل[19]على نتيجة مماثلة, إذ تراجع تركيز الأحماض الدهنية الطيارة الكلية معنويا من 12.24 


$$
\begin{aligned}
& \text { الى } 10.73 \text { مليمول/100 مل نتيجة لإضافة خميرة الخبز بمستوى يجهز عليقة الأبقار بالخميرة الحية بمقدار } \\
& \text { 10 } 10 \\
& \text { الاستنتاجات }
\end{aligned}
$$

نتائج الدراسة الحالية وفرت جملة من الدلائل دفعت الى تلك الاستتتاجات بحصول زيادة في معدل استفادة أبقار الحليب من العناصر الغذائية استجابة لدور الخميرة في تحسين هضم تلك العناصر لاسيما مكونات

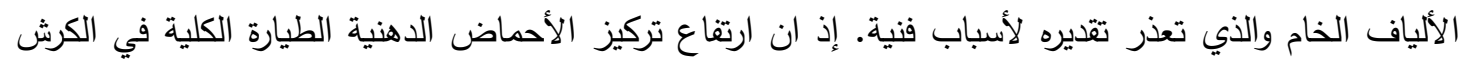
أدى الى زيادة تجهيز أحياء الكرش بالطاقة وبالتالي زيادة النمو والنشاط الميكروبي. وقد ترتب على على ذلتيز ذلك تتامي احتياجات أحياء الكرش من النتروجين مما أدى الى انخفاض تركيز الأمونيا في الكرش. ويمكن أن ترتبط تلكي

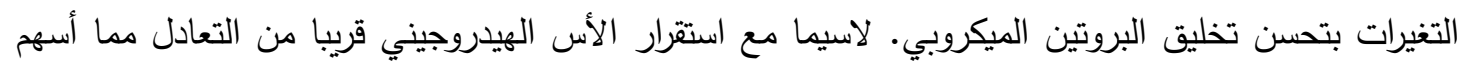
في خلق الظروف الملائمة للنشاط الميكروبي.

يتقدم الباحثان بالشكر لمالك ومدير شركة تاج النهرين/محطة أبقار الديوانية الكبرى والعاملين فيها لتعاونهم البناء في انجاز هذا البحث. والشكر موصول أيضا الى د. يحيى الحسيني/جامعة القاسم الخضراء/كلية الطب البيطري والسيد صلاح مهدي كريم المدرس المساعد/جامعة القادية/كلية الطب البيطري والسيد محمد حمزة أبو اللول لجهودهم في سحب نماذج سائل الكرش والسيد هيثم محمد حسين للمساعدة في تحليل نماذج مرئ المواد العلفية وتقدير خصائص تخمرات السايلج والكرش.

\section{CONFLICT OF INTERESTS.}

There are non-conflicts of interest .

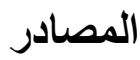

[1] N. Hameed AL- Kudsi and J. Victor Elia, Ministry of Higher Education and Scientific Research, 2010.

[2] A. Ramanathan and K. V. Narasimham, "Comparative evaluation of Saccharomyces cerevisiae and Saccharomyces siccum as a feed supplement on production performance of crossbred cows". probiotics in sustainable food production: Current status and future prospects- Probiotics in Food Production, pp.244-248, 2013.

[3] K. A. Dawson, K. A. Newman and J. A. Boling, "Effect of microbial supplements containing yeast and lactobacilli on roughage-fed ruminal microbial activities", Journal Animal Science, vol. 68, pp.3392-3398, 1990.

[4] M. B. de Ondarza,C. J. Sniffen, L. Dussert, E. Chevaux, J. Sullivan and N. Walker, "Case study: multiple-study analysis of the effect of live yeast on milk yield milk component content and yield and feed efficiency", Prof. Animal Science, vol.26, pp.661-666, 2010.

[5] AOAC. Official Methods of Analysis, Association of Official Analytical Chemists. 18 Ed. Gaithersburg Marlyl and 20877-2417 USA, 2005.

[6] T. Levital, A. F. Mustafaa, P. Seguinb and G. Lefebvrec, "Effects of a propionic acid-based additive on short-term ensiling characteristics of whole plant maize 
and on dairy cow performance", Animal Feed Science Technology, no. 152, pp. 21-32, 2009.

[7] M. Kazemi -Bonchenari, K. Rezayazdi, A. Nikkhah, H. Kohram and M. DehghanBanadaky, "The effects of different levels of sodium caseinate on rumen fermentation pattern, digestibility and microbial protein synthesis of Holstein dairy cows", African Journal Biotechnology, vol.9, pp.1990-1998, 2010.

[8] R. Markham, "A steam distillation apparatus suitable for micro-Kjeldahl analysis Biochem," Journal, vol. 36, pp.790, 1942.

[9] A. Nikkhah, M. D. Bonadaki and A. Zali, "Effects of feeding yeast (Saccharomyces cerevisiae)on production performance of lactating Holstein dairy cow", Iranian Journal Agriculture Science, vol. 35, pp. 53-60, 2004.

[10] L. J. Erasmus, P. H. Robinson, A. Ahmadi, R. Hinders and J. E. Garrett, "Influence of prepartum and postpartum supplementation of a yeast culture and monensin, or both, on ruminal fermentation and performance of multiparous dairy cows", Animal Feed Science and Technology, vol. 122, pp. 219-239, 2005.

[11] M. Dehghan-Banadaky, M. Ebrahimi, R. Motameny and S. R. Heidari, "Effects of live yeast supplementation on mid-lactation dairy cows performances, milk composition, rumen digestion and plasma metabolites during hot season", Journal Applied Animal, vol. 41, no.2, pp. 137-142, 2013.

[12] W. Zhu, Z. Wei, N. Xu, F. Yang, I. Yoon, Y. Chung, J. Liu and J. Wang,“ Effects of Saccharomyces cerevisiae fermentation products on performance and rumen fermentation and micro biota in dairy cows fed a diet containing low quality forage", Journal of Animal Science and Biotechnology, vol. 8, no. 36, pp. 2-9, 2017.

[13] Y. Jiang, I. M. Ogunade, K. G. Arriola, M. Qi, D. Vyas, C. R. Staples and A. T. Adesogan. "Effects of the dose and viability of Saccharomyces cerevisiae. 2. Ruminal fermentation, performance of lactating dairy cows, and correlations between ruminal bacteria abundance and performance measures", Journal Dairy Science, vol. 100, no. 10, pp. 1-17, 2017.

[14] V. Kudrna, K. Poláková, P. Lang and J. Doležal, "The effect of different yeast strains on milk yield, fatty acids profile and physiological parameters in dairy cows", Project No.1G46086, NAVZ, Ministry of Agriculture, The Czech Republic, 2007.

[15] C. M. Guedes, D. M. Gonçalves, A.M. Rodrigues and A. Dias-da-Silva, "Effects of a Saccharomyces cerevisiae yeast on ruminal fermentation and fiber degradation of maize silages in cows", Animal Feed Science and Technology, vol. 145, pp. 27-40, 2008.

[16] P. Doležal, J. Dvořáček, J. Doležal, J. Čermáková, L. Zeman and K. Szwedziak, "Effect of feeding yeast culture on ruminal fermentation and blood indicators of Holstein dairy cows", Acta Veterinary Brno, vol. 80, pp. 139-145, doi:10.2754/avb201180020139, 2011.

[17] D. N. Kamra, L.C. Chaudhary, N. Agarwal, R. Singh and N.N. Pathak, "Growth performance ,nutrient utilization, rumen fermentation and enzyme activities in calves fed on Saccharomyces cerevisiae supplemented diet", Indian Journal Animal Science, vol. 72, pp.472-475,2002.

[18] K.M. Krause, and G.R. Oetzel, "Understanding and preventing subacute ruminal acidosis in dairy herds: are view, Animal Feed Science Technology, vol. 126,pp. 215-236, 2006. 
[19] M. Thrune, A. Bach, M. Ruiz-Moreno, M.D. Stern and J.G. Linn, "Effects of Saccharomyces cerevisiae on ruminal $\mathrm{pH}$ and microbial fermentation in dairy cows: yeast supplementation on rumen fermentation", Livestock Science, vol.124, pp.261-265, 2009.

[20] J.E. Nocek and W.P. Kautz, "Direct-fed microbial supplementation on ruminal digestion, health, and performance of pre- and postpartum dairy cattle", Journal of Dairy Science, vol. 89, pp.260-266, 2006.

[21] F. Rossi, A.D. Luccia, D. Vincenti, and P.S. Cocconcelli, "Effects of peptidic fractions from Saccharomyces Cerevisiae culture on growth and metabolism of the ruminal bacteria Megasphaera elsdenii”, Animal Research, vol. 53,pp.177186, 2004.

[22] F. Chaucheyras-Durand, G. Fonty, G. Bertin, J.M. Salmon, and P. Gouet, "Effects of a strain of Saccharomyces cerevisiae (Levucell SC), a microbial additive for ruminants, on lactate metabolism in vitro", Can. Journal Microbiology,vol.42,pp. 927-933, 1996.

[23] S.C. Martin, and D.J. Nisbet, "Effect of direct-fed microbials on rumen microbial fermentation", Journal Dairy Science, vol.75, pp. 1736-1744, 1992.

[24] A.V. Stella, R. Paratte, L. Valnegri, G. Cigalino, G. Soncini, E. Chevaux, V. Dell'Orto and G. Savoini, "Effect of administration of live Saccharomyces cerevisiae on milk production, milk composition, blood metabolites, and faecal flora in early lactating dairy goats", Small Ruminate Research, vol. 67, pp. 713, 2007.

[25] P. Dolezal, J. Dolezal and J. Trinacty, "The effect of Saccharomyces cerevisiae on ruminal fermentation in dairy cows", Czech Journal Animal Science, vol. 50, no.11, pp. 503-510, 2005.

[26] M. A. Alshaikh, M. Y. Alsiadi, S. M. Zahran, H. H. Mogawer and T. A. Aalshowime," Effect of Feeding Yeast Culture from Different Sources on the Performance of Lactating Holstein Cows in Saudi Arabia", Asian-Aust. Journal Animal Science, vol. 15, no. 3, pp. 352-356,2002.

[27] T. Mašek, Z`. Mikulec, ' H. Valpotic, N. Stojevic', N. Antunac, N. Mikules, Z. Stojevic', N. Filipovic' and S. Pahovic',"Influence of live yeast culture (Saccharomyces cerevisiae) on milk production and composition, and blood biochemistry of grazing dairy ewes during the milking period", Act. Vet. Brno, vol. 77, pp.547-554, 2008.

[28] S.R. Heidari Khormizi, M. Dehghan- Banadaky, K. Rezayazdi and A. Zali, "Effect of live yeast and Aspergillus niger meal supplementation on milk yield, feed efficiency and nutrients digestibility in Holstein lactating cows", Journal of Animal and Veterinary Advances, vol.14, pp.1934 -1939, 2010.

[29] H.M. Khattab, F.A. Salem, M.M. Sayeda and H.M. Nagh, "Effect of YeaSacc, Lacto-Sacc supplementation and energy level on performance, rumen activity, some rumen activity, some blood constituents and carcass traits in growing sheep", Egypt Journal Nutr. Feed, vol. 4, pp.981-989, 2003.

[30] J.P. Jouany, "Dvacet let výzkumu kvasinkových kultur a jejich masivní nástup v současné době ve výživě přežvýkavců," In: Sbor. 15. evropského přednáškového turné firmy Alltech, Brno, pp. 29-39, 2001.

[31] L. Majdoub-Mathlouthi, K. Kraiem and M. Larbier, "Effects of feeding Saccharomyces cerevisiae Sc 47 to dairy cows on milk yield and milk components, in Tunisian conditions", Livestock Research for Rural Development, vol. 21, no. 5, 2009. 
[32] P. H. Robinson, "Yeast products for growing and lactating ruminants: a literature summary of impacts on rumen fermentation and performance", Cooperative Extension, University of California - Davis, Davis. CA. 2009. [Online]. Available:

http://animalscience.ucdavis.edu/faculty/robinson/Articles/FullText/Web200901 .pdf.

[33] C. J. Newbold, R. J. Wallace and F. M. McIntosh, "Mode of action of the yeast Saccharomyces cerevisiaeas a feed additive for ruminants", Br. J. Nutr. vol. 76, pp. 249-261, 1996.

[34] M. Doreau, and J. Jouany, "Effect of a Saccharomyces cerevisiae on nutrient digestion in lactating dairy cows", journal dairy science, vol. 81, pp. 3214 3221, 1998.

[35] H. M. Sullivan, and S. H. Martin, "Effects of Saccharomyces cerevisiae culture on in vitro mixed ruminal microorganism fermentation", Journal Dairy Science, vol. 82, pp. 2011-2016, 1999.

[36] R. G. S. Bruno, H. M. Rutigliano, R. L. Cerri, P. H. Robinson and J. E. P. Santos, "Effect of feeding Saccharomyces cerevisiae on performance of dairy cows during summer heat stress", Animal Feed Science and Technology, vol. 150, pp. $175-186,2009$.

[37] R. E. Hungate, The rumen and its microbes. Academic Press, Inc., New York, NY. (1966)

[38] E. N. Bergman, "Energy contributions of volatile fatty acids from the gastrointestinal tract in various species", Physiological Reviews, vol. 70, no. 2, pp. 567-590, 1990.

[39] B. Kowalik, J. Skomiał, J. J. Pająk, M. Taciak, M. Majewska, and G. Bełżecki, "Population of ciliates, rumen fermentation indicators and biochemical parameters of blood serum in heifers fed diets supplemented with yeast (Saccharomyces cerevisiae) preparation", Animal Science Papers and Reports, vol. 30, pp. 329-338, 2012.

[40] C. D. Lu, J. R. Kawas, and O. G. Mahgoub, "Fiber digestion and utilization in goats”, Small Ruminate Research, vol. 60, pp.45-52, 2005.

[41] B. Mwenya, B. Santoso, C. Sar, B. Pen, R. Morikawa, K.Takaura, K.Umetsu, K. Kimur and J. Takhashi, "Effect of yeas culture and galacto-oligosaccharides on ruminal fermentation in Holstein cows," Journal of Dairy Science, vol.88, pp. 1404-1412, 2005.

[42] P. E. V. Williams and C. J. Newbold, "Rumen Probiosis: the effect of novel microorganisms on rumen fermentation and ruminant productivity", In: Recent Advances in Animal Nutrition pp. 211-227,1990. Butterworth London UK.. 